\title{
Story creation and cognitive biases
}

\author{
Jun Nakamura \\ Faculty of Global Management, Chuo University, Higashinakano 742-1 \\ Hachioji-shi, Tokyo 192-0393, Japan* \\ E-mail: jyulis.77f@g.chuo-u.ac.jp \\ www. Global.chuo-u.ac.jp/English/
}

\begin{abstract}
A wide variety of stories can be created from the same material. The author asked several teams to create stories, focusing on cognitive biases. As expected, a variety of stories were created by each team, and cognitive biases were identified in the process of creating them. In other words, if it is assumed that cognitive bias is a driving force behind the creator's intentions, then it can be concluded that the creator must have a certain strong intention when creating a story.
\end{abstract}

Keywords: Story, cognitive bias, analogy, and creativity

\section{Introduction}

Studies of "creativity" are difficult to replicate. This research has sometimes been said to be inherently dubious. However, human prosperity is nothing but the accumulation of creativity derived not only from destructive innovation but also from gradual creative innovation that makes it sustainable.

The word "creativity" can be used to describe a wide range of products, depending on what one is creating. The product may be an object, a service, or a new technology. Whatever the case, to attract people, it is important to tell a "narrative story", and I believe that an attractive story makes a deep impression on people and inspires them to remember it.

I have been studying the process of designing and generating stories. In doing so, I have focused on how the characteristics of stories change depending on the presence or absence of external stimuli (Nakamura, 2020). This paper is focused, first, on the effects of availability heuristics, termed cognitive biases in this paper, such as the selective use of memory. I explore the process by which different teams given the same data set create different stories.

The second chapter touches on related research and experimental tools. Next, Chapter 3 describes stories' impression points and items for assessing cognitive biases. This is followed by Chapter 4, which introduces an experiment based on participants' self-assessment. The results of the experiment are then presented in Chapter 5. This is followed by a discussion aimed at analyzing and interpreting the results of the experiment, and the final chapter offers a conclusion.

\section{Related research and experimental tool}

As an area of research in support of activities to create stories, the aim was to develop games that enhance composition and creativity to reconstruct combinations of words and thereby create new concepts. The importance of the meaning and role of words in the design process, which is a creative activity, has been noted (Nakakoji and Yamamoto, 2010). The game 
developed is aimed at connecting words by analogy (Holyoak and Thagard, 1996) and discovering new expressions in various combinations. It is called the Analogy Game (Nakamura and Ohsawa, 2007).

This type of game falls into the category of "serious games" in the broadest sense of the word. Serious games are defined as "games used to solve problems in education and other areas of society" (Sawyer 2001). Examples would include training in firefighting activities, emergency and medical training, and language learning. Overseas, the use of serious games to address the issue of the educational use of games has been attracting attention (Fujimoto, 2006). Since this analogy game was aimed at fostering composition and creativity, it is of a type that players explore and structure by themselves (Quinn, Beattie, McNaught, and Wills, 1994).

The analogy game is introduced as an experimental tool in the following manner. At the beginning, players are given 20 word cards that have been pre-classified (panel A in Figure 2). The word cards are clustered into several categories that are given relevant concept names commonly used in daily life, such as "fruits", which would cover bananas, strawberries, and melons. In this paper, such concepts are regarded as ready-made concepts.

After the game begins, players attempt to dismantle the ready-made concepts and reconstruct new combinations by moving the word cards around (Figure $2 \mathrm{~B})$. Words that are grouped together at this stage are highlighted in the same color. This is because the system recognizes groups by their color code. Players then generate new concept names for the reconstructed groups. If one word card is left out of these groups, the composition must be reviewed again. The game ends when all of the word cards are classified into one of the new groups (Figure $2 \mathrm{C}$ ). For example, suppose that services and medical care were categorized as "businesses", sushi and baseball as "Japan", and cars and barbecue as "family travel". Players could recombine these ready-made concepts by combining service, medical, and barbecue into "compassion" and by bundling sushi, baseball, and cars into "overseas expansion", thereby creating concepts that are completely different from the original concept.

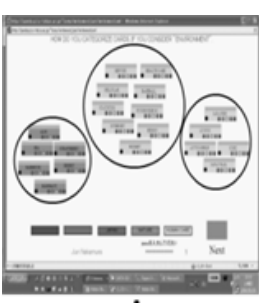

A

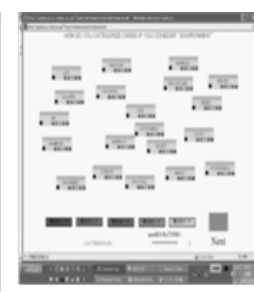

B

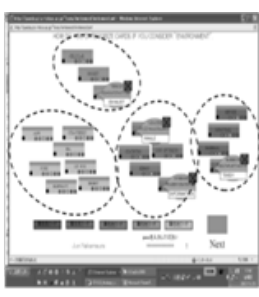

C
Fig. 1. Flow of the Analogy Game

\section{Story impression points and assessment}

The aim of the experimental tool mentioned in the previous chapter was to support creative activities (Hori, 2007). The present chapter explains impression points in story creation as a deliverable self-assessment through debriefing after the game.

\subsection{Story impression points}

It has been suggested that creativity can be evaluated from the viewpoints of originality and novelty as well as practicality and appropriateness (Paletz and Peng, 2008). Therefore, the author concluded that "Innovative" and "Feasible" are appropriate as impression points for created stories. In addition, three impression points, Usefulness, Scale expansion, and Barrier to rival, were added, with reference to the value proposition design proposed by Imazu ${ }^{\dagger}$ (2016), which excluded quantitative evaluation points. Therefore, the following five items were identified as the impression points for created stories in this paper: Innovation, Feasibility, Usefulness, Scale expansion, and Barrier to rival.

\subsection{Assessment}

Next, the assessment items for cognitive biases are introduced. In relation to cognitive biases, self-awareness is metacognition (Brown, 1978). This paper aimed to use metacognition to explore the source of story creation. For this purpose, as described in the first chapter, the participants were asked to self-assess relevant characteristics, considering the availability heuristics such as people's selective use of memory, as forms of cognitive bias. In this case, what are the types of cognitive bias? Bazerman and Moore identified 11 types of bias that can occur during decision making (Baserman 
and Moore, 2013). In this paper, four of these were selected for consideration in creative activities, excluding quantitative items.

- Ease of Recall: cognitive bias that occurs toward easily recalled material based on the clarity of memory

- Retrievability: cognitive bias toward an object to be remembered that is structurally easy to retrieve

- Confirmation trap: cognitive bias based on the assumption that one's own hypothesis is correct

- Anchoring effect: cognitive bias toward available information as a starting point and tendency not to move far beyond that anchor

Based on the above, the assessment items for cognitive bias were organized in this experiment as follows.

Assessment item 1 (memory versus hypothesis)

$1-\mathrm{X}=$ Recalling someone's memories of past events affected your ideas

$1-\mathrm{Y}=$ Not your memories but your team's own tentative hypothesis affected your ideas

Assessment item 2 (stuck to the cards versus took off from cards)

$2-\mathrm{X}=$ You were affected by the meaning of a given set of cards and continued to stick with that until the end $2-\mathrm{Y}=$ You were influenced by a given set of cards, but the idea took off from that

Assessment item 3 (not shared versus shared)

$3-\mathrm{X}=$ During the team discussion, a unique idea arose that was not shared by other individuals

$3-Y=$ The ideas were sufficient using only the information shared among team members

Assessment item 1-X was combined with Ease of recall and Retrievability because both were types of cognitive bias related to ones' memory of the past. On the other hand, assessment item 1-Y asked the participants whether they felt a cognitive bias suggesting that they believe their hypothesis is correct as a confirmation trap or as a memory of the past.

Assessment item 2-X asked whether the participants recognized the anchoring effect, which is a type of cognitive bias, and assessment item 2-Y asked whether they recognized the expansion of their ideas without their being anchored to a given card.

Assessment item 3 was not a straightforward question of cognitive bias, but it asked for "bounded awareness" in conducting teamwork. In this experiment, the participants spent more than 2 hours in teamwork, in effect, and the amount of information in the conversation was considerable. In sorting this information during the discussion, the participants had to process unconsciously. In the post-experimental review, assessment item 3-X asked participants whether they felt that the information that they considered useful (i.e., the cognitive bias of the confirmation trap was also present here) was ignored or overlooked. In contrast to 3-X, assessment item 3-Y asked whether the respondents felt that the information that they found useful was sufficiently shared and agreed upon within the team.

\section{Experiment}

In this paper, we conducted an experiment using the analogy game introduced in Chapter 2 in the following manner:

Experiment date: Friday, November 27, 2020

Participants: Twenty-one second-year students of the Faculty of Global Management, Chuo University

Experimental method: Twenty-one people were divided into six teams and presented with a set of word cards on the screen as follows. Capital letters in the following indicate the names of the clusters, and lower-case letters indicate individual words on the cards.

- TRIP ADVISOR: discount ticket, foreign tour, backpacker, guide, word of mouth

- FACEBOOK: search, friends, like!, share, network

- IKEA LIFE: living room, do it yourself, easy to store, Europe, wardrobe

- STARBACKS: yen400, third place, extra job, coffee, steamer

The above set of word cards is the same as that used in our previous experiment (Nakamura, 2020). The difference between the previous experiment and the present one is that this was an experiment not with one team but with six teams. Participants were instructed to devise a story connecting several newly reconstructed 
clusters (a set of word cards) using the experimental tool. The story was to be expressed in PowerPoint using a copy of the screen created with the experimental tool. The story was then presented to the participants. After the presentation of the story, participants were asked to do the following two tasks:

- Give a score to stories created by teams other than your own, taking into account the impression points mentioned in Chapter 3. A total of 20 points was the upper limit of the score. However, the same number of evaluation points should not be given to more than one team.

- Assess what cognitive biases were present in response to your own team using the assessment items described in Chapter 3. Participants were instructed to answer questions $\mathrm{X}$ and $\mathrm{Y}$ for each of the assessment items $(1,2$, and 3$)$, for a total of 10 points, and determine the ratio of $\mathrm{X}$ and $\mathrm{Y}$ that they think applies to them.

\section{Results}

Even though the word cards provided in the experiment were the same for all teams, different teams created different stories. The impression point scores for the six teams are shown in Figure 2, with team 4 receiving the highest rating.

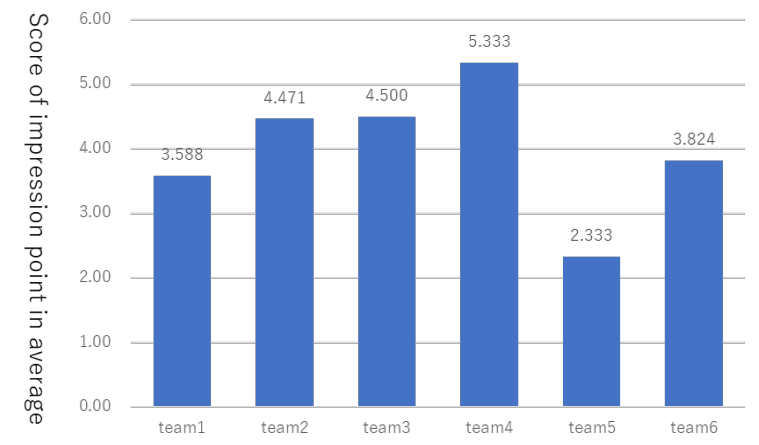

Fig. 2. Impression points rating by team

The team 4 story that received the best ratings was the following:

Story theme. Four things that the students on Working Holiday need might be Cheap clothes, Information, Relax and Tour.
Story. Since they are working in foreign countries to earn money, study a language, and learn the culture at the same time, it is better to save clothing money and travel around if they have time. To save money on their clothing, it would be better for them to purchase or borrow seasonal clothes at a sales market or garage sale. Also, since it will be difficult to get information in foreign countries, where they do not have friends or family, they might need to use an online community to get various kinds of information during their working holidays. Finally, it is important to relax after work every day.

An interesting slide from the team 4 presentation is shown in Figure 3.

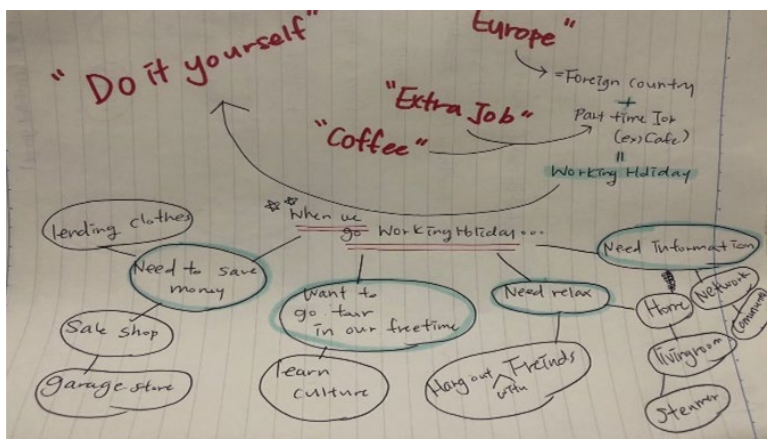

Fig. 3. A slide representing the thinking process

This is a picture of a note on the thinking process underlying creating a story. The note was written down and projected onto one of the presentation slides. The explanation given for this slide was as follows:

(1) The first four words that stood out to me were, "Do it yourself", "coffee", "extra-job”, and "Europe". I decided to connect the four.

(2) Think of it this way, it's a working holiday! That is said, a part-time job in a foreign country is indeed a working holiday!

(3) On a working holiday, you have to do it all by yourself.

(4) As a mini-story derived from the above, on a working holiday we need to save money, we might want to go on a tour in our free time, we might need to relax, and we might need information.

The results of the self-assessment from all of participants are shown in Table 1. 
Table 1. Self-assessment of cognitive biases.

\begin{tabular}{|c|c:c:c:c:c:c||c:c|}
\hline & Team1 & Team2 & Team3 & Team4 & Team5 & Team6 & AVE & STD-EVP \\
\hline $1-X$ & 3.00 & 4.25 & 7.00 & 3.00 & 2.67 & 0.50 & 3.40 & 1.96 \\
\hline $1-Y$ & 7.00 & 5.75 & 3.00 & 7.00 & 7.33 & 9.50 & 6.60 & 1.96 \\
\hline $2-X$ & 3.00 & 4.25 & 5.33 & 2.67 & 5.00 & 5.50 & 4.29 & 1.11 \\
\hline $2-Y$ & 7.00 & 5.75 & 4.67 & 7.33 & 5.00 & 4.50 & 5.71 & 1.11 \\
\hline $3-X$ & 0.75 & 4.00 & 2.67 & 2.00 & 4.67 & 0.25 & 2.39 & 1.60 \\
\hline $3-Y$ & 9.25 & 6.00 & 7.33 & 8.00 & 5.33 & 9.75 & 7.61 & 1.60 \\
\hline
\end{tabular}

\section{Discussion}

Focusing on teams 4 and 3, which had the highest ratings, what kind of cognitive biases did the team members feel? In team 4, the most remarkable assessment item that the team member reported was item $2-Y$, which was unique compared to the other teams. This indicates that they did not stick to the given key words until the end, and they perceived themselves to have made a leap in the meaning of the words. This may be due to the so-called polysemy effect, in which a given key word has multiple meanings (Costello and Keane, 2000). It could be said that blending a meaning that is different from the original meaning (Fauconnier, 1994) produced a creative story. In fact, if we look at the thought process of team 4 , as depicted in Figure 3, the approach to the mini-story that connects the first four key words (Europe, Extra-Job, Coffee, and Do it yourself) was noteworthy. From this mini-story, the team expanded the idea and connected the remaining key words to form the whole story.

The second most highly rated team was team 3 , which, like team 4, showed characteristic cognitive biases that were different from the other teams. Team 3 had a higher rating on item $1-\mathrm{X}$ than the other teams, i.e., they felt that their memories were more effective in generating stories than were their own free ideas and hypotheses. The possible reasons for this are discussed below.

In the case of team 3, it might be presumed that memory was given priority because the consensus was that teamwork must take precedence over mutually agreed-upon ideas, resulting in the hypotheses of various participants cancelling each other out. However, team 4, the most highly rated team, gave the opposite answer. That is, team 4 responded with a free hypothesis that was not anchored in their own memory. This contrast is quite interesting. Given that teams 4 and 3 did not respond uniquely to the question, it is difficult to figure out the story-generating algorithm. However, at least from the above results, we can say that the cognitive biases of assessment items 1 and 2 , whether $\mathrm{X}$ or $\mathrm{Y}$, are characteristically different to those of the other teams (i.e., representing highest or lowest awareness).

Now, we discuss assessment item 3 . For both team 3 and team 4, assessment item Y was ranked higher than item $\mathrm{X}$. This implies that both teams seemed to be in a good environment for the members to share their opinions with each other. On the other hand, for team 5, which had the lowest score, $\mathrm{X}$ was higher than $\mathrm{Y}$, indicating that individual opinions were not reflected. That means that no chemical reaction yielding new ideas occurred in the mix of different opinions from the team members.

\section{Conclusions}

Cognitive biases were evident throughout the experiment in the process of creating stories. In other words, if we assume that cognitive biases are the driving force behind the creator's intentions, then it is essential that the creator have a certain strong intention to yield a memorable story.

The experiment in this paper, however, is just a case study of a limited number of people, and there are still many works to be addressed before the findings can be generalized. In the future, the author intends to focus more on the process of creating stories.

\section{Acknowledgement}

This study was carried out with the cooperation of undergraduate students of Chuo University, Japan. Also, the Analogy Game was upgraded and developed further with the support of Mr. Tano, President of Expert Gig Co., Ltd. This research was supported by Kakenhi (JSPS KAKENHI Grants Numbers 17K03872 and 19K03062).

The English in this document has been checked by at least two professional editors, both native speakers of English. For a certificate, please see: http://www. textcheck. com/certificate/rTtVeD.

\section{References}

1. J. Nakamura, Effect of external activate factors serving as clue: creating new products or services ideas with storytelling, T. Ogata and J. Ono (eds.), Bridging the Gap Between AI, Cognitive Science, and Narratology With Narrative Generation, IGI Global, Hershey, PA, 2020. 
2. K. Nakakoji and Y. Yamamoto, Wording as external representations to interact with: its role and nature throughout a design process, Cognitive Studies: Bulletin of the Japanese Cognitive Science Society, 17(3), 2010, pp. 474-490.

3. K.J. Holyoak and P. Thagard, Mental Leaps, MIT Press, MA, 1996.

4. J. Nakamura and Y. Ohsawa, The visualization of concept creation throughout the analogy game, Proceedings of the $2^{\text {nd }}$ Oukan Conference, Transdisciplinary Federation of Science and Technology, 2007, pp. 157-160.

5. B. Sawyer, Serious Games: Improving Public Policy through Game based Learning and Simulation, in Foresight and Governance Project, Woodrow Wilson International Center for Scholars, Publication 2002-1, retrieved on Dec.5, 2020, at https://www.scribd.com/document/38259791/SeriousGames-Improving-Public-Policy-through-GamebasedLearning-and-Simulation.

6. T. Fujimoto (2006) Kaigai niokeru serious game no kanousei (in Japanese), in Proceedings of the Conference on Simulation \& Gaming, Autumn, 2006.

7. C. N. Quinn, K. Beattie, C. McNaught, S. Wills, Designing educational computer games, Proceedings of Interactive Multimedia in University Education: Designing for Change in Teaching and Leadership IFIP Transactions, 1994, pp. 45-47.

8. K. Hori, Creativity Support, Theory and Application of AI Approach toward Creativity, Ohmusha, Tokyo, 2007.

9. S. B. Paletz and K. Peng, Implicit theories of creativity across cultures, Journal of Cross-Cultural Psychology, 39(3), 2008, pp. 286-302.

10. M. Imazu, Hashirinagara kangaeru sinkijigyou no kyoukasho (in Japanese), Kanki Pub., Tokyo, 2016.

11. A. L. Brown, Knowing when, where, and how to remember: a problem of metacognition, in R. Glaser, (Ed.), Advances in Instructional Psychology, Lawrence Erlbaum Associates, Hillsdale, NJ, 1978.

12. M. H. Bazerman and D.A. Moore, Judgement in Managerial Decision Making, John Wiley \& Sons, Inc., MA, 2013.

13. F. J. Costello and M. T. Keane, Efficient creativity: constraint-guided conceptual combination, Cognitive Science, 24(2), 2000, pp. 299-349.

14. G. Fauconnier, Mental Spaces, Cambridge University Press, Cambridge, UK, 2008. 